\title{
Critical Velocities for High Speed Particle Deposition in Kinetic Spraying
}

\author{
Jingwei $\mathrm{Wu}^{1,2}$, Hongyuan Fang ${ }^{1}$, Sanghoon Yoon $^{2}$, Changhee Lee ${ }^{2, *}$ and HyungJun Kim ${ }^{3}$ \\ ${ }^{1}$ State Key Lab of Advanced Welding Production Technology, Harbin Institute of Technology, \\ 150001, Harbin, Heilongjiang, P. R. China \\ ${ }^{2}$ Kinetic Spray Laboratory (NRL), Division of Materials Science \& Engineering, Hanyang University, 17 Haengdang-dong, \\ Seongdong-Ku, Seoul 133-791, Seoul, South Korea \\ ${ }^{3}$ Welding Research Center, Research Institute of Industrial Science \& Technology, Pohang, South Korea
}

\begin{abstract}
During kinetic spraying (or cold gas dynamic spraying), small solid particle can be deposited onto the substrate by a supersonic velocity impaction. A critical velocity of flying particle was found for the minimum deposition speed. Generally, it was thought that higher impact velocity caused higher deposition efficiency. However, some different phenomena were observed during spraying processing in laboratory.

In this study, individual particle impact tests have been carried out to probe the effect of impact velocity. Instead of deposition efficiency (DE) of coatings, ratio of bonds, which was defined as the number fraction of attached particles to total impact particles (Craters + Bonds) in unit area of impact surface, was measured in our test. A maximum velocity for particle deposition was observed, because of which a high fraction of rebounded particles occurred at a high impact velocity. A model considering the adhesion and rebound energy was built up to estimate the particle/substrate interaction. The particle deposition behavior was a result of the competition between adhesion and rebound energies during the impact process. Only when the adhesion energy was higher than the rebound energy, the impacting particles could be attached onto the substrate. [doi:10.2320/matertrans.47.1723]
\end{abstract}

(Received November 30, 2005; Accepted June 1, 2006; Published July 15, 2006)

Keywords: kinetic spraying, cold gas dynamic spraying, individual particle impact, deposition

\section{Introduction}

Kinetic spraying (or cold gas dynamic spraying) occurs when small solid particles are accelerated to supersonic velocity, and then impacted them onto a substrate. In the kinetic spraying processes, dense coatings are produced without any significant heating of the spray powder and substrate material. The kinetic energy of the particles plays a central role in the impaction and deformation behaviors. Because of the advantages realized at a low process temperature, including a low oxidation of the powder and substrate and a high-rate of material deposition, the kinetic spraying technique has developed quickly over the last decade. Kinetic spraying was first developed in the mid-1980s at the Institute of Theoretical and Applied Mechanics of the Siberian Division of the Russian Academy of Science in Novosibirsk. ${ }^{1-3)}$ Further research has been conducted in the USA and Germany, and a few years ago a system of kinetic spraying was developed for industrial applications and laboratory research. ${ }^{4-7)}$ In kinetic spraying applications developed to date, a wide range of pure metals, metal alloys, polymers, composites and nano-materials have been successfully deposited onto a variety of substrate materials.

The critical particle velocity is accepted as a method of characterizing this spray technique. Numerous studies ${ }^{7-9)}$ show that particles require a minimum velocity to be deposited onto the substrate and also suggest that higher impact velocities yield coatings with high bond strength and lower porosity. Based on computer modeling, Hamid and Frank, et al. ${ }^{7}$ provide an equation for estimating the critical velocity, which is a function of the properties of feedstock material, such as density, melting point, ultimate strength and initial particle temperature. Individual particle impact be-

*Corresponding author. E-mail: chlee@ hanyang.ac.kr haviors are the basis of research into kinetic spray coating. Many numerical and experimental studies have been done to reveal the impact process of single particles. In Alkhimov et al.'s investigations, ${ }^{10-12)}$ the deformation, attachment, and contact temperature of individual particles are discussed in detail. The surface micrographs of pure aluminum impacted onto different substrates are presented in Zhang's study. ${ }^{13)}$ In numerical studies, Kurochkin et al. ${ }^{14)}$ develop the equation of Shorshorov and Kharlamov ${ }^{15)}$ from D-Gun spraying and apply it to kinetic spraying, which can then estimate the relative strength of the bond between the particles and the substrate. Further modeling was done by Papyrin et al. ${ }^{8)}$ that considers elastic recovering during the impact process and presents a model of individual particle deposition using adhesion and elastic energies. In this model, the particle can be deposited onto the substrate after its velocity is higher than the critical velocity, which is the result of the competing adhesion and elastic energies. However, there is lack of the information about individual particle impact and deposition behaviors over a large range of impact velocities. Generally, it is thought that a higher impact velocity results in increased deposition efficiency. ${ }^{8,9)}$ But in our experiments, something different was observed. The present study attempted to clarify rebound phenomenon during the impact of individual soft particles onto a hard substrate. The energy model of the impacting process was discussed and the calculation result was used to explain the rebound phenomenon.

\section{Experimental Procedures}

\subsection{Spraying system}

In this study, a commercially available CGT kinetic spraying system was used. The equipment and the coating process are described in detail in the literature. ${ }^{4-6)}$ A de Laval type nozzle with a converging/diverging inner form was used 


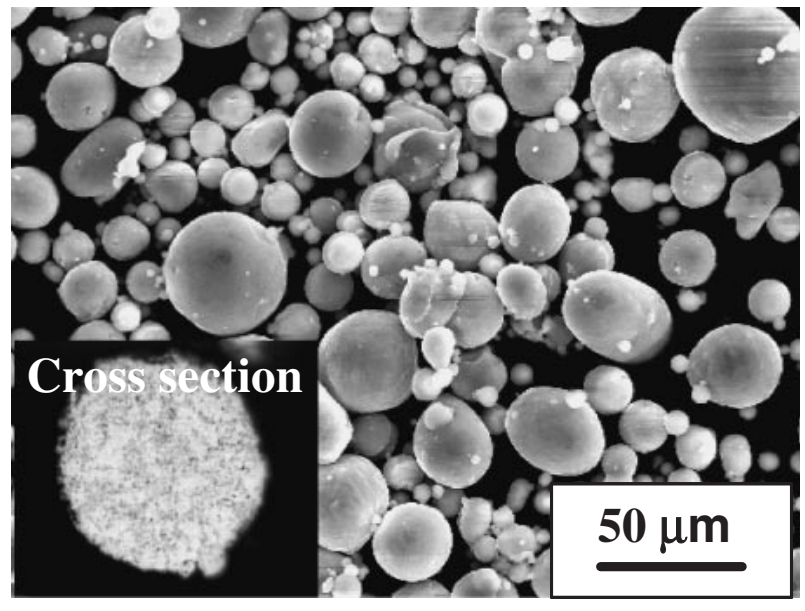

Fig. 1 Feedstock: Al-Si(wt $\% 78: 12)$, spherical, $+5 \mu \mathrm{m}-45 \mu \mathrm{m}$ (mean size $25 \mu \mathrm{m})$.

(Standard type nozzle of CGT kinetic spraying system, the diameter ratio of throat and exit is ${ }^{4,16,17)}$ ). In order to obtain a wide range particle velocities, nitrogen and helium gas were used for the process gas and the carrier gas. The process gas pressure ranged from 0.3 and $3.0 \mathrm{MPa}$ and the temperature included $300,400 \mathrm{~d}, 500^{\circ} \mathrm{C}$. The flow quantity of carrier gas was set as $8 \%$ of the nitrogen process gas and $10 \%$ of the helium process gas. The feedstock was Al-12Si powder with a mean particle size of $25 \mu \mathrm{m}$ and a density of $2.66 \mathrm{~g} / \mathrm{cm}^{3}$, as shown in Fig. 1. In order to observe individual particle behavior, a low feed rate was used $(<1.0 \mathrm{~g} / \mathrm{min})$. Mild steel was used for substrates, the surface of which was polished as smooth as a metal mirror $\left(<1.5 \mu \mathrm{m} R_{\mathrm{a}}\right)$. The target substrate was fixed at $30 \mathrm{~mm}$ in front of the exit of nozzle. The gun scanned with a speed of $0.3 \mathrm{~m} / \mathrm{s}$ during coating experiments.

\subsection{Analysis}

To measure the in-flight velocity of the particles, the SprayWatch system (Oseir Ltd, Finland) was used. During the experiments, track images of the flying particles were taken using a high-speed camera. Using the particle flying distance and camera exposure time, the particle velocity could be calculated. Although the particle size, the distance to the exit of the nozzle, the radial position and other factors do affect particle velocity, only the mean particle velocity was used in this study, which came from large numbers (>300) of measured flying particles, to simplify the analysis,. The surfaces of coating specimens with different impact velocities were observed by Scanning Electron Microscopy (SEM, including Energy Dispersive Spectroscopy, EDS), by which the forms of the attached particles and the craters on the substrate were investigated and quantified.

\section{Results and Discussion}

\subsection{Rebound phenomenon}

Individual particles of high velocity impacted onto the substrate, and a single particle deposition could be obtained, as shown in Fig. 2. Besides the bonded particles, numerous craters were distributed on the substrate surface. To estimate the efficiency of particle deposition, the numbers of craters

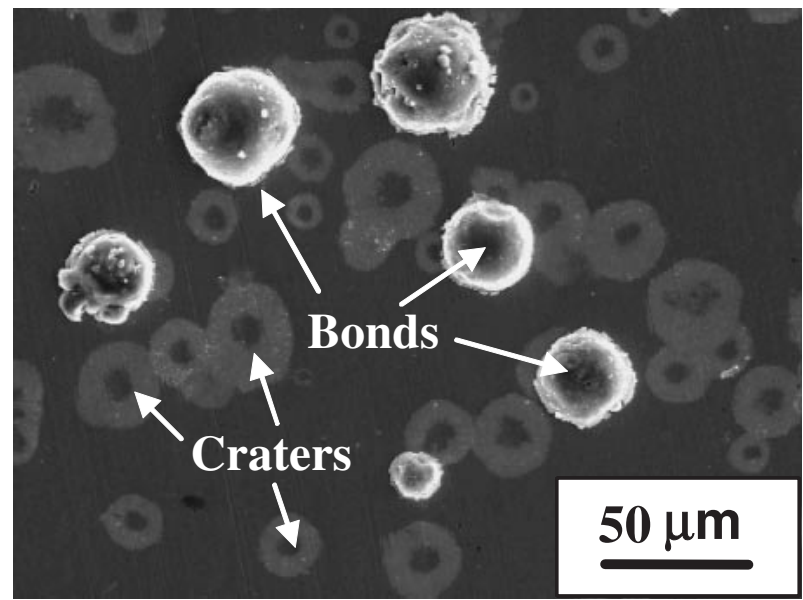

Fig. 2 SEM micrographs of the contact surfaces (top view, mean particle velocity of $500 \mathrm{~m} / \mathrm{s}$, process gas temperature $400^{\circ} \mathrm{C}$ ).

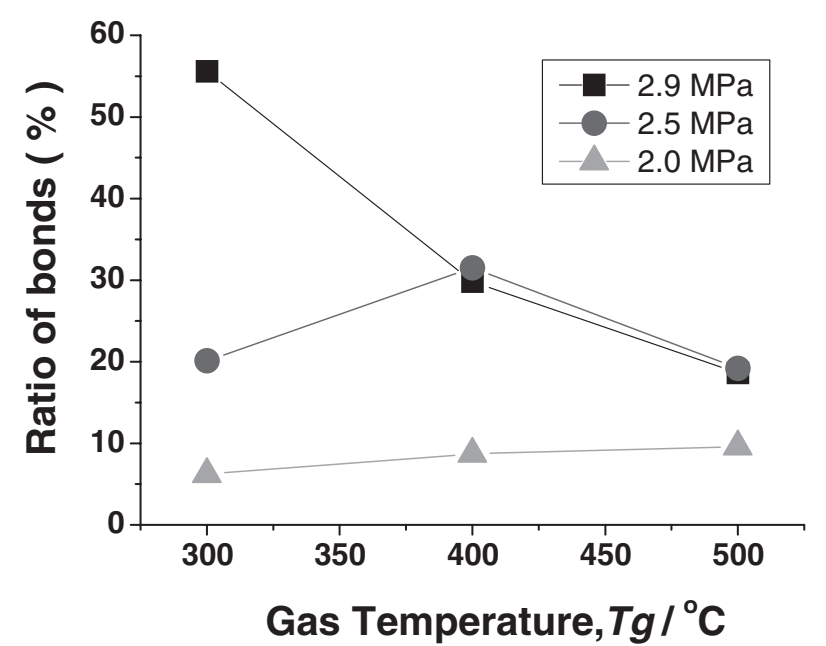

Fig. 3 The effect of process gas (nitrogen) temperature on the ratio of bonds for an Al-Si feedstock impacting onto a mild steel substrate at different gas pressure.

and attached particles were counted. Deposition efficiency (DE), which is the weight fraction of deposited coating divided by the total fed, is normally used to value the coating efficiency in thermal and kinetic spraying. Instead of the deposition efficiency of coatings, the ratio of bonds, which is defined as the fraction of attached particles to total impacted particles (Craters + Bonds) in a unit area of impact surface, was used in the individual particle impact test. In our experiments, the different process gas temperature and pressure consisting of different types of gases (nitrogen and helium) were used to control the impact particle velocity. Figure 3 shows the ratio of bonds at varied gas conditions. It surprised us that the effects of the process gas temperature on ratio of bonds were out of accord at each process gas pressure. Ratio of bonds increased with the process gas temperature at the process gas pressure of $2.0 \mathrm{MPa}$, while it decreased at the pressure of $2.9 \mathrm{MPa}$. When the process gas pressure was 2.5 $\mathrm{MPa}$, the ratio of bonds increased from the process gas temperature of 300 to $400^{\circ} \mathrm{C}$, and decreased from the process gas temperature of to $500^{\circ} \mathrm{C}$. In kinetic spraying, the particle 


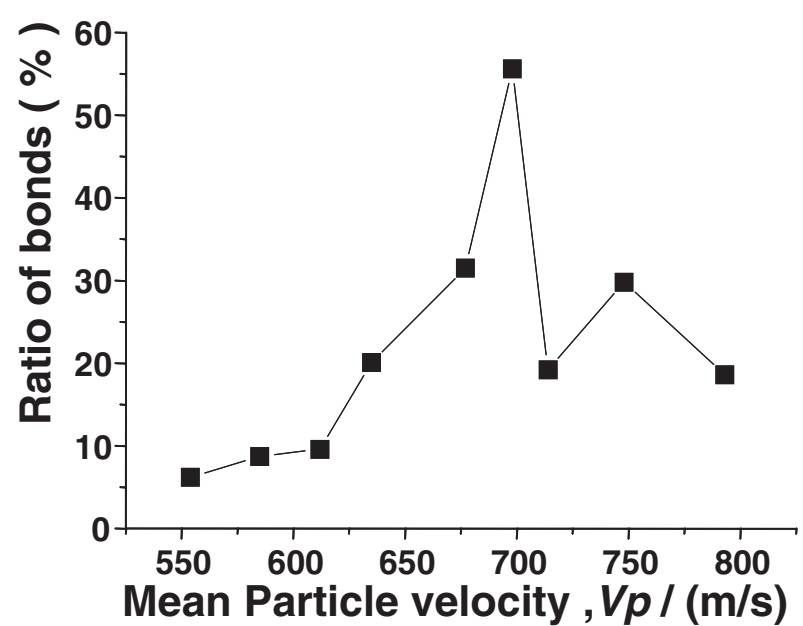

Fig. 4 The ratio of bonds for an Al-Si feedstock impacting onto a mild steel substrate when nitrogen is used as the process gas at pre-heat temperature of $300-500^{\circ} \mathrm{C}$.

velocity increases with the process gas temperature and pressure. So the results allowed us deduce that the ratio of bonds was not always growing with particle velocity.

To clear that, the measured data of ratio of bonds were rearrangement with the mean particle velocity, which was measured in our experiments. In Fig. 4, the ratio of bonds increased with the particle velocity first, and after got a maximum value it dropped with further increasing the particle velocity. However, the curve in Fig. 4 is not smooth and convictive, because that the gas temperature not only affected the particle velocity also acted on particle temperature before impact, which should be important to particle deposition. To remove the effect of process gas temperature (or particle temperature), in our further experiments, the process gas temperature was fixed at $400^{\circ} \mathrm{C}$, and the process gas pressure consisting of different types of gases (nitrogen and helium) were used to control the impact particle velocity. After tests with large range of particle velocity, the results were shown in Fig. 5, in which the bond ratio started at about $250 \mathrm{~m} / \mathrm{s}$ and increased to close to $70 \%$ at $700 \mathrm{~m} / \mathrm{s}$, and then decreased with further increases in impact velocity. We called this the "Rebound Phenomenon". In our experiments, mean particle velocities of higher than $800 \mathrm{~m} / \mathrm{s}$ were obtained by using helium as the process gas when the process gas temperature was fixed at $400^{\circ} \mathrm{C}$. The result indicated two critical velocities were found during the high-speed impacting deposition with large range of particle velocity: one was the minimum critical velocity for particle deposition onto the substrate; another was the critical velocity for particle rebound from the substrate at a higher impact velocity.

\subsection{Rebound energy}

To approach the impact behavior and particle/substrate interaction, a energy model including rebound energy and adhesion energy was used to explain the rebound phenomenon, which was developed firstly by Alkhimov, ${ }^{10-12)}$ Kurochkin, ${ }^{14)}$ and Papyrin. ${ }^{8)}$

Following the static and dynamic indentation tests, ${ }^{15)}$ the impact process consisted of an elastic-plastic loading followed by an elastic unloading during which the elastic

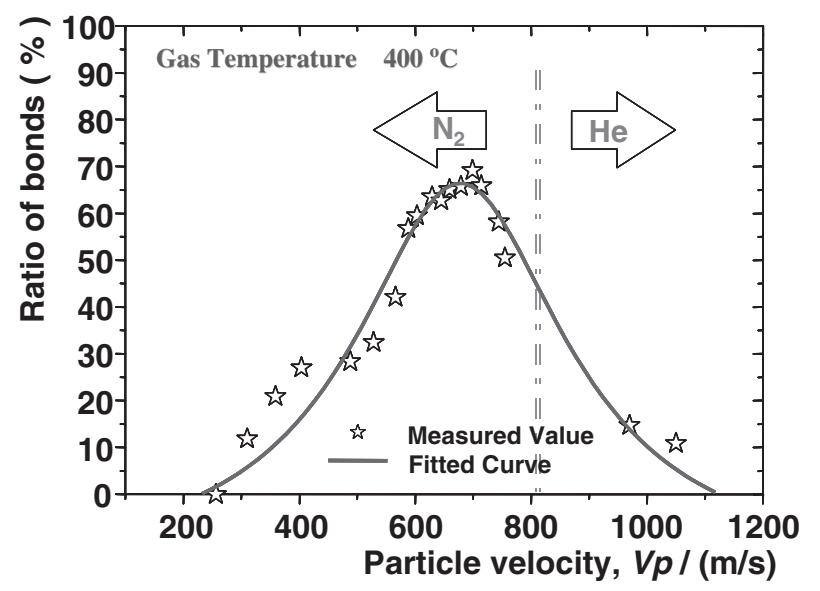

Fig. 5 The ratio of bonds for an Al-Si feedstock impacting onto a mild steel substrate when nitrogen and helium are used as the process gas at preheat temperature of $400^{\circ} \mathrm{C}$.

recovery occurs. The energy required for bouncing the particle from the substrate during the unloading moment is defined as rebound energy in the impact process of kinetic spraying. Referring to the drop-ball dynamic hardness test, ${ }^{18,19)}$ the rebound energy $(R)$ is expressed as:

$$
R=\frac{1}{2} e_{r} m_{p} V_{p}^{2}
$$

where the recoil coefficient $e_{r}$ for spherical particles is given by:

$$
e_{r}=11.47\left(\frac{\bar{\sigma}_{Y}}{E^{*}}\right)\left(\frac{\rho_{p} V_{p}^{2}}{\bar{\sigma}_{Y}}\right)^{-\frac{1}{4}}
$$

Here, $m_{p}$ and $V_{p}$ are the mass and velocity of impact particle, $\bar{\sigma}_{Y}$ is the effective yield stress during the impact, $E^{*}$ is the conventional elastic modulus of the feedstock and substrate materials, and $\rho_{p}$ is the density of the particle material. Note that shockwave effects and adiabatic heating can complicate the impact process. A strain-hardening, strain-rate sensitive, thermal-softening, and deformation localization must be considered for the calculation of the effective yield stress $\bar{\sigma}_{Y}$, which is provided from the Johnson-Cook plasticity model. ${ }^{20,21)}$

\subsection{Adhesion energy}

Adhesion behaviors during supersonic impaction are important for particle deposition and coating build-up in kinetic or cold spray technologies. The process of adhesive interaction is considered to be one of bond generation between the particle and substrate during contact. The adhesion energy $(A)$, which is defined as the energy for detaching the bonded particle from the substrate, is expressed as: $A=a \% A_{\max }$, where $A_{\max }$ is the maximum adhesion energy of a given particle to the substrate; $a \%$ is the fraction of bonded atoms per unit adhesive interface, and is also called the relative strength of the bond between the particle and substrate. In previous research that investigated interactions during high speed thermal spraying (D-Gun Spraying), Shorshorov and Kharlamov ${ }^{15)}$ developed a relation for the fraction of bonded atoms during high speed impact. 
Kurochkin, et al. ${ }^{14)}$ improve this relation for the kinetic spraying process and report the following expression:

$$
a \%=1-\exp \left\{-v t_{c} \exp \left[\frac{-E_{a}}{k T_{c}+\left(1-e_{r}\right) m_{a} V_{p}^{2} / 2}\right]\right\}
$$

where $v$ is the natural frequency of eigen-oscillations of atoms in the crystal lattice, $t_{c}$ is the contact time, $E_{a}$ is the activation energy of the chemical bonds $\left(E_{a}=0.5 \times 10^{-19} \mathrm{~J}\right.$ for pure aluminum and $E_{a}=1.55 \times 10^{-19} \mathrm{~J}$ for iron $\left.{ }^{7)}\right), T_{c}$ is the contact temperature, $k$ is the Boltzmann constant, $e_{r}$ is the recoil coefficient during elastic recovery (provided by eq. (2)), $m_{a}$ is the atomic mass of the impact particle, and $v_{p}$ is the velocity of the impact particle. In eq. (3), $a \%$ is mainly affected by the contact temperature $T_{c}$ and impact velocity $v_{p}$.

The maximum adhesion energy can be expressed as: $A_{\max }=S_{c} N_{a} E_{1}$. Here $S_{c}$ is the contact area of a single particle to the substrate; $N_{a}$ is the total number of atoms in the unit contact plane. This number can be estimated using the crystal lattice parameter $a\left(N_{a}=\frac{4}{\sqrt{3} a^{2}}\right.$ for the close-packed face of FCC lattice). $E_{1}$ is the energy of a single bond between two atoms and it is evaluated by sublimation energy.

\subsection{The competition of rebound and adhesion energy}

In the framework of particle deposition in thermal and kinetic spraying processes, the particle is assumed to attach onto the substrate when the adhesion energy $(A)$ is higher than the rebound energy $(R)$. The comparison of the adhesion energy to the rebound energy of individual particle impact is shown in Fig. 4. When the particle velocity was low, the adhesion energy was lower than the rebound energy, and then the particles can not be deposited. When the particle velocity achieved a threshold value (200-300 m/s), the adhesion energy started to increase. When the particle velocity was high enough for the adhesion energy to exceed the rebound energy, particle deposition was obtained. Here the particle velocity where the curves of two energies intersect was the critical velocity for individual particle deposition onto the substrate, which is marked with an " $x$ " in Fig. 6. In our calculations, a second point (marked with a " $\triangle$ ") where the energy curves intersect was obtained, which is called the maximum velocity for the particle deposition onto the substrate. When the particle velocity was higher than this point, the adhesion energy was below the rebound energy and the impacted particle could not attach onto the substrate. The critical and maximum velocities can be defined as the minimum and maximum velocities for individual particle deposition onto the substrate by impaction. For an assemblage of given particle and substrate, only the particle whose velocity is between the critical and maximum velocities can be attached onto the surface of the substrate by kinetic impinging. This accounted for the rebound phenomenon observed in the individual particle impact tests shown in Figs. 4 and 5.

Figure 7 shows the critical and maximum velocities for different particle diameters. It can be observed that the critical velocity was unlimited on particle size while the maximum velocity decreased notably with the increasing of particle size. The impact velocity for deposition must be

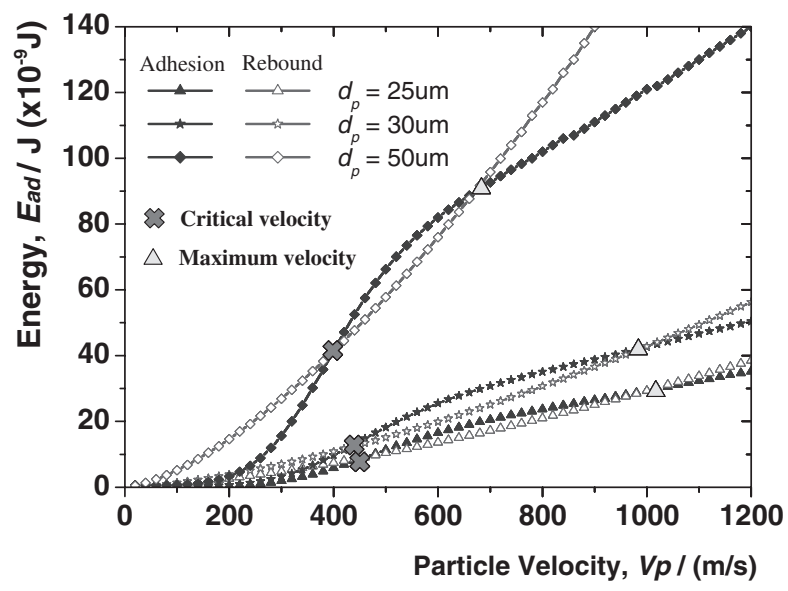

Fig. 6 Calculated adhesion energy and rebound energy for various sized Al-Si feedstock particles impacting onto a mild steel substrate.

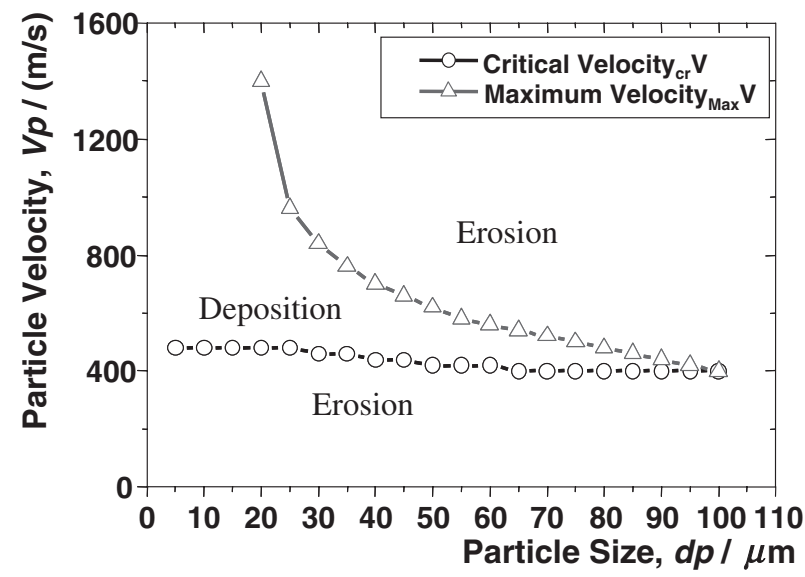

Fig. 7 Calculated critical velocity and maximum velocity for varisized AlSi feedstock deposition onto the mild steel substrate.

higher than critical velocity but lower than maximum velocity. So the figure of particle velocity-particle size can be divided into three regions. The region between critical velocity and maximum lines was deposition region, and the other regions (lower than critical velocity or higher than maximum velocity) were just the erosion regions, where particle could not be deposited onto the substrate and only made a short-peening. One thing worthy of note was that the maximum velocity for a big enough particle became as low as the critical velocity and the deposition region disappeared. That is to say very big particle could not be deposited onto the substrate with any impact velocities. So a critical particle size existed for deposition. This is a deduction from our modeling, in which a plastic particle impacting onto an undeformed substrate is the presupposition. However, whether the maximum velocity and the critical particle size are adaptive to other feedstock and substrate assemblies, more experiments and studies are needed.

In individual particle impact process, the deposition characteristic is the result of the competition between the adhesion energy and rebound energy, which are affected by the impact conditions (temperature and velocity) and the material properties of the feedstock and substrate. Occur- 
rence of rebound phenomenon is also dependent on the material properties of the feedstock and substrate. When the adhesion energy and rebound energy for a certain particle and substrate combination never meet, this particle can not be deposited onto the substrate at any impact velocity. When the two curves meet once, the particle could bond onto the substrate after it gets the critical velocity for its deposition. When the adhesion and rebound energy meet more than once, the rebound phenomenon could be observed. Because of the rebound phenomenon, a certain range of impact velocities exists for optimum deposition.

\section{Conclusions}

In this study, an individual particle impact test has been carried out. From observation of the impact surface, a rebound phenomenon was observed such that a high particle velocity caused a high rebound particle fraction during individual particle impact. Based on a plastic particle impacting onto an undeformed substrate, a model about the particle/substrate interaction was built up in order to probe into high speed particle impact and deposition behaviors. From the numerical modeling, the deposition of individual particles was controlled by the adhesion energy and the rebound (elastic recovering) energy. Only when the adhesion energy was higher than the rebound energy, the impacting particles could be attached onto the substrate. Besides a critical (minimum) velocity, a maximum impact velocity was found for the particle deposition onto the substrate. The maximum velocity decreased with the increasing of particle size, which indicated that the particle with a limited size can be deposited onto a substrate by high speed impact.

\section{Acknowledgements}

This work was financially supported by MOCIE (Ministry of Commerce Industry and Energy) under the project named "development of advanced surface modification technologies based on welding" and the Korea Science and Engineering Foundation (KOSEF) through the National Research Laboratory (NRL) program.

\section{REFERENCES}

1) A. P. Alkhimov, V. F. Kosarev and A. N. Sov. Paryrin: Phys. Dokl. 35 (1990) 1047.

2) A. P. Alkhimov, V. F. Kosarev and A. N. Sov. Paryrin: American Inst. of Phys.: (1991).

3) A. P. Alkhimov, A. N. Paryrin, V. F. Kosarev, N. J. Nesterovich and M. M. Shuspanov: Gas-dynamic spray method for applying a coating. US Patent 5,302,414, April 12, 1994.

4) W. Kroemmer, P. Heinrich and P. Richter: Int. Therm. Spray Conf., (Orlando 5-8 may 2003), (ASM Int., Materials Park, OH, U.S.A., $2003)$ pp. 97.

5) R. E. Blose, T. J. Roemer, A. J. Mayer, D. E. Beatty and A. N. Papyrin: Int. Therm. Spray Conf., (Orlando 5-8 may 2003), (ASM Int., Materials Park, OH, U.S.A., 2003.) pp. 103.

6) J. Karthikeyan and C. M. Kay: Int. Therm. Spray Conf., (Orlando, 5-8 may 2003), (ASM Int., Materials Park, OH, U.S.A., 2003.) pp. 117.

7) H. Assadi, F. Gärtner, T. Stoltenhoff and H. Kreye: Acta Mater. 51 (2003) 4379-4394.

8) A. N. Paryrin, S. V. Klinkov and V. F. Kosarev: Int. Therm. Spray Conf., (Orlando 5-8 may 2003), (ASM Int., Materials Park, OH, U.S.A., 2003) pp. 27.

9) M. Grujicic, J. R. Saylor, D. E. Beasley, W. S. DeRosset and D. Helfritch: Applied Surface Science 219 (2003) 219-211.

10) A. P. Alkhimov, S. V. Klinkov and V. F. Kosarev: Physical Mesomechanics (2000) 31-53.

11) A. P. Alkhimov, A. I. Gudilov, V. F. Kosarev and N. I. Nesterovich: Journal of Applied Mechanics and Technical Physics 41 (2000) 188.

12) A. P. Alkhimov, S. V. Klinkov and V. F. Kosarev: Journal of Applied Mechanics and Technical Physics 41 (2000) 245

13) D. Zhang, P. H. Shipway and D. G. McCartney: Int. Therm. Spray Conf., (Orlando 5-8 may 2003), (ASM Int., Materials Park, OH, U.S.A., 2003) pp. 45.

14) Y. V. Kurochkin, Y. N. Demin and S. I. Soldatenknov: Chemical and Petroleum Engineering 38 (2002) 245.

15) M. K. Shorshorov and Y. A. Kharlamov: Physical and chemical principles of detonation gas spray coating. ed. by Nauka, (Moscow, Russia, 1978).

16) A. C. Fischer-Cripps: Vaccum 58 (2000) 569.

17) A. C. Fischer-Cripps: Nanoindentation. (Springer Verlag New York 2002) pp. 8.

18) W. Kohlhöfer and R. K. Penny: Int. J. Pres. Ves. \& Piping. 61 (1995) $60-65$.

19) R. B. Clough, S. C. Webb and R. W. Armstrong: Mater. Sci. Eng. A. (2003) 360-396.

20) J. F. Molinari and M. Ortiz: International Journal of Impact Engineering. 27 (2002) 347.

21) M. Grujicic, C. L. Zhao, W. S. DeRosset and D. Helfritch: Materials and Design. 25 (2004) 681. 